\title{
Higher risk of cardiovascular events
}

Patients with one or more kidney stones have an increased risk of cardiovascular (CV) events, according to a new study by Todd Alexander and colleagues from the University of Alberta, Canada.

The link between kidney stones and CV risk factors, including hypertension, diabetes, dyslipidaemia and chronic kidney disease, is well recognized; however, evidence linking kidney stones to specific CV events is limited, with studies providing conflicting results. The researchers used the Alberta Kidney Disease Network database to assemble a cohort of 3,195,452 adults $\geq 18$ years (median follow-up 11 years), excluding patients with end-stage renal disease owing to their increased risk of $\mathrm{CV}$ disease. Incidence of kidney stones and CV outcomes were assessed using validated algorithms applied to physician claims and hospital administrative data.

Overall, 25,532 (0.8\%) and 91,465 (3\%) patients had at least one kidney stone or CV event, respectively, during follow-up. Compared with patients without kidney stones, patients with one or more kidney stones had a markedly increased risk of myocardial infarction (primary end point), even after adjusting for $\mathrm{CV}$ and other confounding risk factors (HR 1.40). Kidney stones were also associated with an increased risk of cardiac revascularization procedures (HR 1.63) and stroke (HR 1.26).

The results suggest that kidney stones are a causative factor in future $\mathrm{CV}$ events. The authors speculate that the underlying pathophysiology leading to kidney stones, formed largely due to calcium precipitations, might also mediate coronary artery and intracranial calcification, potentially explaining the increased risk of CV events and stroke observed in patients with kidney stones.

\section{David Holmes}

Original article Alexander, R. T. et al. Kidney stones and cardiovascular events: a cohort study. Clin. J. Am. Soc. Nephrol. doi:10.2215/CJN.04960513 